\title{
Percepções de Alunos dos Anos Finais do Ensino Fundamental Sobre o Uso das Tecnologias Digitais nas Atividades de Sala de Aula
}

\section{Camila Maria Bandeira Scheunemann ${ }^{1}$, Caroline Medeiros Martins de Almeida ${ }^{2}$, Paulo Tadeu Campos Lopes ${ }^{1}$}

${ }^{1}$ Programa de Pós-graduação em Ensino de Ciências e Matemática - Universidade Luterana do Brasil (ULBRA)

Avenida Farroupilha, 8001- Bairro São José - Canoas - RS - Brasil

${ }^{2}$ Programa de Pós-graduação em Gestão Educacional - Universidade do Vale do Rio dos Sinos (UNISINOS)

Avenida Nilo Peçanha, 1600 - Bairro Boa Vista - Porto Alegre- RS - Brasil

camila.b91@hotmail.com, carolinemalmeida@unisinos.br,pclopes@ulbra.br

\begin{abstract}
This study aimed to investigate and analyze the perceptions of students in the final years of elementary school on the use of digital technologies in classroom activities. Participants were 80 students from a municipal school in Sapucaia do Sul/RS. Data were collected through a questionnaire with open and closed questions, analyzed by content analysis and the scores of the answers. The results showed that teachers rarely use digital technologies and the most common resource is the PowerPoint. Students were open to the use of technologies and would like to use them during classes, preferably through applications and games and believe that they can facilitate teaching and learning, by providing a more fun class, facilitating practices and increasing your interest.
\end{abstract}

Resumo. Este estudo teve por objetivo investigar e analisar as percepções de alunos dos anos finais do Ensino Fundamental sobre o uso de tecnologias digitais nas atividades de sala de aula. Os participantes foram 80 alunos de uma escola municipal de Sapucaia do Sul/RS. Os dados foram coletados por questionário com questões abertas e fechadas, analisadas pela análise de conteúdo e pelos escores das respostas. Os resultados apontaram que os professores utilizam pouco as tecnologias digitais, de forma rara e o recurso mais usual é o PowerPoint. Os alunos apresentaram-se abertos ao uso das tecnologias e gostariam de utilizá-las durante as aulas, preferencialmente por meio de aplicativos e jogos, e acreditam que elas podem facilitar o ensino e a aprendizagem, por proporcionar uma aula mais divertida, facilitar práticas e aumentar seu interesse.

\section{Introdução}

As tecnologias digitais (TD) têm sido introduzidas de forma crescente nos espaços da sociedade; no âmbito escolar, apesar de ainda em andamento, sua inserção tem ocorrido de forma ascendente, tendo em vista o aperfeiçoamento das práticas pedagógicas do contexto digital contemporâneo. 
Os discentes têm as TD presentes em seu cotidiano, as quais apresentam alternativas e potencialidades variadas de aplicação no campo educacional, o que impulsiona a elaboração de propostas educativas que as considerem, em prol de aulas mais interessantes e contemporâneas [Moran 2012; Moran, Masetto e Behrens 2013; Almeida, Costa e Lopes 2017].

O público que frequenta os anos finais do Ensino Fundamental, essencialmente jovens, em sua maioria, possuem um contato cotidiano com as TD, nos espaços internos e externos das instituições escolares. Neste sentido, considera-se útil aproveitar estes recursos que já são trazidos para o âmbito escolar pelos estudantes, mas que poucas vezes são utilizados em sala de aula para fins pedagógicos.

A inserção das tecnologias na esfera educativa provoca transformações na maneira de ensinar e aprender, modificando os processos tradicionais de ensino, antes mais passivos, para agora, ativos [Machado 2016]. Neste contexto, Gewehr e Strohschoen (2017) apontam que os alunos do Ensino Fundamental são receptivos às potencialidades que as tecnologias apresentam, as quais têm se mostrado desafiadoras e convidativas nas situações de ensino e aprendizagem nas quais estão presentes.

Encontra-se na literatura alguns estudos que buscaram investigar as percepções de alunos sobre a utilização das TD nas atividades de sala de aula [Löbler, Pretto e Bolzan 2013; Machado 2016; Gewehr e Strohschoen 2017]; no entanto, pesquisas para o levantamento destas percepções com alunos dos anos finais do Ensino Fundamental são menos frequentes. Por isso, este trabalho pretende contribuir para elucidar o que pensam estes estudantes sobre a temática e como seu uso ocorre em seu cotidiano em sala de aula.

Considerando este contexto, a pergunta que norteou este estudo foi: Quais as percepções de alunos dos anos finais do Ensino Fundamental sobre a utilização de tecnologias digitais nas atividades de sala de aula? Nesta perspectiva, teve por objetivo investigar e analisar as percepções de alunos dos anos finais do Ensino Fundamental sobre o uso de tecnologias digitais nas atividades de sala de aula.

\section{Tecnologias Digitais no ensino e aprendizagem em atividades de sala de aula}

O cenário contemporâneo modificou-se com a inserção das TD, impactando a forma como as pessoas trabalham, estudam e seus hábitos de vida; como resultado, isso trouxe inúmeros desafios e novas necessidades nas mais variadas áreas do conhecimento [Morais, Morais e Góis 2018].

Um número considerável de alunos utiliza cotidianamente as TD, por isso, inseri-las nas atividades escolares pode auxiliar no ensino e aprendizagem, contribuindo para que os discentes possam desenvolver a autorregulação, aproveitando a mídia digital para estimular situações de ensino [Almeida, Santos e Lopes 2018].

Diante disso, existe uma situação dicotômica, pois de um lado estão os discentes instigados pelas tecnologias e que esperam por aulas mais dinâmicas e inovadoras, e de outro, os professores enfrentam o desafio da atualização constante de seus saberes relacionados com as tecnologias aplicadas ao ensino e aprendizagem [Silva e Nascimento 2020]. 
Os recursos digitais oferecem aos docentes e discentes possibilidades de aumentar o acesso a informações atualizadas, produções científicas a nível mundial, desenvolver autoaprendizagem, estratégias inovadoras de elaboração de atividades, integrando as diversas mídias existentes (como vídeo, imagem e som) [Masetto 2013].

Fuza e Miranda (2020) discutem sobre a transversalidade das TD presente na Base Nacional Comum Curricular (BNCC), temática já mencionada em documentos oficiais anteriores, como os Parâmetros Curriculares Nacionais, e que se encontra em destaque, agora na BNCC, havendo neste último uma intensificação sobre a importância do digital, o que necessita um intenso repensar sobre o uso destes recursos em sala de aula.

No entanto, é indispensável o entendimento de que apenas inserir as TD não é suficiente para melhorar o ensino e a aprendizagem, é necessário que este uso contribua para o desenvolvimento do aluno [Oliveira, Mello e Franco 2020]. Santos, Rodrigues e Junior (2018) corroboram, admitindo que apenas incluir as tecnologias não garante melhorias aos processos educativos, sendo fundamental, portanto, que os docentes sejam capazes de atuar de maneira crítica e criativa com o uso destes recursos.

\subsection{Revisão de estudos empíricos}

Gewehr e Strohschoen (2017) analisaram as percepções de alunos do Ensino Fundamental sobre a utilização das tecnologias nas estratégias empregadas por seus professores; os discentes ressaltaram que se sentem mais envolvidos e consideram que as práticas são mais eficazes quando as tecnologias estão presentes.

Em seu estudo, Backes e Pavan (2014) identificaram os efeitos das identidades dos estudantes da educação básica em razão da cultura digital, percebidos pelos professores. Verificaram, através das análises apontadas pelos docentes, que existem características prejudiciais ao processo de ensino e aprendizagem, como desinteresse, distração, dispersão e comodismo; características favoráveis, como curiosidade, interesse, criatividade e interlocução; e pode haver também uma mescla dos dois tipos.

Löbler, Pretto e Bolzan (2013) investigaram as percepções de 83 estudantes do Ensino Médio de escolas públicas sobre o uso das tecnologias no apoio à aprendizagem e verificaram uma recepção positiva dos discentes sobre esta utilização, que acreditam que elas trazem um impacto positivo nos relacionamentos entre alunos $\mathrm{e}$ alunos/professores.

Machado (2016) realizou uma pesquisa com discentes de um curso Técnico de Informática a fim de relatar a experiência com o emprego das tecnologias no ensino e verificar as percepções dos estudantes sobre utilizá-las; como resultado, aponta que os jovens participantes acreditam na importância das tecnologias e ressaltaram seu gosto pelas aulas interativas com o auxílio de recursos como vídeos e tutoriais online.

$\mathrm{Na}$ atualidade, continua sendo um desafio para os professores e pesquisadores desenvolver possibilidades cada vez mais assertivas para melhorar o impacto das TD nos processos educativos, valendo-se delas como parte importante das formas de aprendizado. Assim, as TD podem ser introduzidas em todos os níveis de ensino, para atividades variadas e na entrega de conteúdo, a fim de obter todos os benefícios que podem proporcionar [Sarker et al. 2019]. 


\section{Metodologia}

Esta pesquisa é de natureza exploratória, que de acordo com Piovesan e Temporini (1995, p. 321) é aquela feita "como estudo preliminar, realizado com a finalidade de melhor adequar o instrumento de medida à realidade que se pretende conhecer". Ainda, trata-se de um estudo quantitativo-qualitativo, que visa a utilização destes dois métodos de forma a se evitar as fragilidades de cada um deles, em separado [Kirschbaum 2013].

Os participantes foram 80 alunos de uma escola municipal de Ensino Fundamental do município de Sapucaia do Sul/RS, sendo o total de discentes dos anos finais da instituição. A referida escola é de pequeno porte, com apenas seis salas de aula, funciona no período da manhã e tarde, atendendo duas modalidades de ensino: a Educação Infantil e o Ensino Fundamental.

Os dados foram coletados no segundo semestre de 2019, por meio da aplicação de um questionário com perguntas abertas e fechadas, as quais são apresentadas nos resultados. Os dados das perguntas fechadas foram analisados por meio dos escores das respostas e, naquelas abertas, por meio da análise de conteúdo, com base em Bardin (2011).

A análise de conteúdo abrange um grupo de processos que visam a descrição do conteúdo da essência de mensagens, que possibilitam a posterior inferência sobre elas [Bardin 2011]. Na análise de conteúdo realizada, o corpus (material textual a ser analisado) foi constituído pelas perguntas e respostas dos alunos, e a categorização ocorreu de forma a posteriori, ou seja, as categorias foram estabelecidas a partir das respostas dos participantes e não de forma anterior à análise.

Para cada pergunta aberta ou que requeria justificativa, foi elaborado um quadro com a categorização correspondente. Cabe esclarecer que, nos quadros apresentados, o número de respostas (f) pode ultrapassar o número de alunos participantes, o que ocorre em função de uma mesma resposta estar enquadrada em mais de uma subcategoria.

\section{Resultados e Discussão}

Quanto à caracterização, os participantes foram 80 alunos, sendo 43 meninas e 37 meninos, com idades entre 11 e 16 anos, cursando do sexto ao nono ano do Ensino Fundamental.

Inicialmente, foi perguntado aos discentes sobre o uso das TD por parte de seus professores nas aulas, e, posteriormente, sobre o que os alunos pensam em relação à sua utilização e preferências pelas TD.

$\mathrm{Na}$ primeira pergunta foram questionados: "Os professores utilizam propostas educativas que envolvam as TD nas aulas?" Para esta questão, 77 alunos $(96,2 \%)$ responderam que não e apenas três $(3,8 \%)$ responderam que sim. Este dado mostra que, na realidade destes estudantes, as TD são pouco utilizadas em práticas pedagógicas em sala de aula. Resultado semelhante foi encontrado na pesquisa de Gewehr e Strohschoen (2017), na qual os alunos de Ensino Fundamental disseram constatar pouco uso de tecnologias nas aulas.

Os participantes que disseram "Sim" na primeira pergunta deveriam responder a pergunta 2, uma questão filtro sobre a frequência desta utilização das TD pelos professores; para esta questão, os três alunos que responderam de forma afirmativa na 
pergunta anterior, relataram que a utilização ocorria de forma rara. Schuhmacher, Filho e Schuhmacher (2017) discutem sobre as barreiras e obstáculos enfrentados pelos docentes quanto ao uso das tecnologias na prática pedagógica e argumentam que o professor, muitas vezes, vem de uma geração com uma realidade construída de forma diferente daquela do aluno, ou que pode ter tido experiências desagradáveis com o uso das tecnologias, fatores que dificultam sua inserção nas estratégias de sala de aula.

Ainda, os estudantes que marcaram a opção "Sim" na questão um, precisavam responder a segunda questão filtro (questão 3), que pedia quais eram as TD que estes professores utilizavam, com as opções: PowerPoint, jogos, blogs e aplicativos. Para esta pergunta, todos os três alunos responderam que a única TD utilizada era o PowerPoint. Para Panucci-Filho, Santos e Brito (2011) existem divergências a respeito da utilização do PowerPoint nas práticas de ensino, o que abre margem para discussões sobre seu uso, e que este recurso necessita cuidados quanto à adoção única.

Além da utilização das TD pelos seus professores, os discentes foram solicitados a responder sobre sua própria percepção quanto ao uso destes recursos para o ensino e aprendizagem. Na pergunta 4 foram questionados: "Você gostaria de utilizar as TD nas aulas? Justifique." Para este questionamento, todos os 80 alunos (100\%) responderam de forma afirmativa, demonstrando uma grande abertura ao uso das TD. As justificativas apresentadas foram categorizadas e estão apresentadas no Quadro 1:

Quadro 1. Justificativas dos alunos sobre o uso de Tecnologias Digitais (TD) nas aulas

\begin{tabular}{|c|c|c|c|}
\hline \multirow{2}{*}{$\begin{array}{c}\text { CATEGORIA } \\
\text { Gostaria de utilizar } \\
\text { TD nas aulas }\end{array}$} & SUBCATEGORIAS PRIMÁRIAS & f & $\%$ \\
\cline { 2 - 4 } & Sem justificativa & 38 & 46,9 \\
\cline { 2 - 4 } & Por ser legal & 9 & 11,1 \\
\cline { 2 - 4 } & Melhorar o aprendizado & 5 & 6,2 \\
\cline { 2 - 4 } & Seria algo diferente & 4 & 6,2 \\
\cline { 2 - 4 } & Ajudaria nas aulas & 4 & 4,9 \\
\cline { 2 - 4 } & Aulas mais práticas & 3 & 3,7 \\
\cline { 2 - 4 } & Seria mais interessante de aprender & 3 & 3,7 \\
\cline { 2 - 4 } & Gosto de utilizar & 2 & 2,4 \\
\cline { 2 - 4 } & Facilitaria o ensino & 2 & 2,4 \\
\cline { 2 - 4 } & Pesquisa em aula & 2 & 2,4 \\
\cline { 2 - 4 } & Jogos são legais & 1 & 1,3 \\
\cline { 2 - 4 } & Seria uma boa opção & 1 & 1,3 \\
\cline { 2 - 4 } & Sou familiarizado & 1 & 1,3 \\
\hline \multirow{2}{*}{ Total } & Para estudar & 1,3 \\
\hline
\end{tabular}

Com base no Quadro 1 observa-se que, apesar de expressarem sua disposição em utilizar as TD em aula, a maioria (38 alunos, 46,9\%) não justificou o porquê. Entre os que justificaram, as maiores frequências foram "Por ser legal" ( $f=9,11,1 \%)$, "Melhorar o aprendizado" ( $\mathrm{f}=5,6,2 \%)$ e "Seria algo diferente" ( $\mathrm{f}=5,6,2 \%)$. Lopes, 
Almeida e Costa (2014) mencionam que os computadores, celulares, internet, softwares e jogos são recursos que os alunos, em sua maioria, utilizam cotidianamente desde cedo, com os quais já crescem familiarizados.

Para Anastacio e Voelzke (2020), a utilização das TD em âmbito educativo apresenta-se como uma possibilidade eficaz que deve ser levada em conta no ensino, pois estimula os discentes na construção de suas aprendizagens. Desta forma, estes recursos podem ajudar o professor no engajamento dos estudantes e, por isso, torna-se importante sua inserção nas práticas pedagógicas.

Os discentes foram questionados, na pergunta 5, sobre qual tipo de tecnologias iriam preferir em sala de aula, entre as opções: PowerPoint, jogos, blogs e aplicativos. O resultado está expresso na Figura 1:

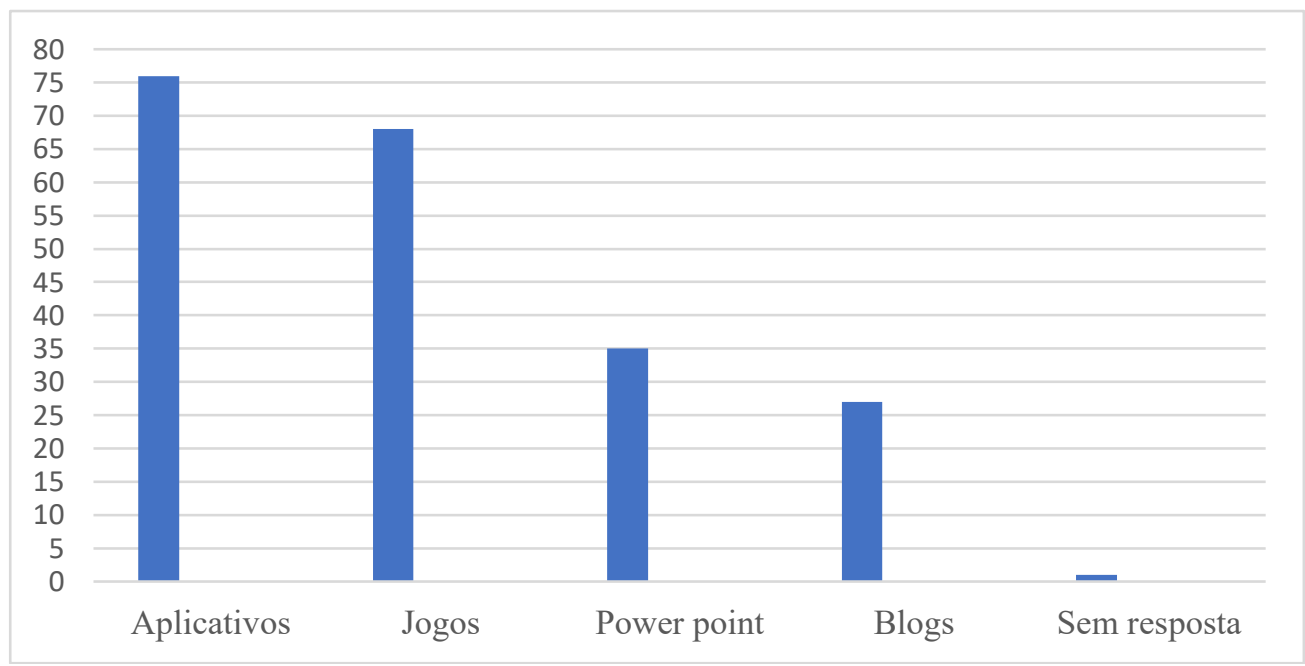

Figura 1. Tipos de Tecnologias Digitais (TD) que os alunos preferem.

A Figura 1 mostra que a maioria dos discentes (76) diz preferir os aplicativos, seguido pelos jogos (68); já o PowerPoint e os blogs ficaram com uma preferência menor, especialmente o blog.

Os aplicativos apresentam-se como uma possibilidade de recurso no ensino, pois ajudam o estudante a desenvolver uma aprendizagem menos abstrata, e apresentam os conteúdos de maneira mais ilustrativa [Almeida, Lopes e Lopes 2015]. Os jogos estão cada vez mais presentes no dia a dia dos jovens, e despertam seu interesse, especialmente pelos desafios que propõem [Pery, Cardoso e Nunes 2010].

O uso didático dos recursos digitais ajuda a promover uma postura mais ativa dos alunos, permitindo abandonar a passividade e envolver-se mais como construtores do conhecimento e protagonistas de suas aprendizagens [Gomes, Martinho e Carneiro 2016].

Os alunos foram questionados, na pergunta 6: "Achas que as TD podem facilitar o processo de ensino e aprendizagem? Justifique". Para esta pergunta, 78 alunos responderam "Sim", um aluno "Não" e um que "Mais ou menos". As justificativas foram categorizadas e estão apresentadas no Quadro 2: 
Quadro 2. Uso das Tecnologias Digitais (TD) no ensino e aprendizagem, justificativas

\begin{tabular}{|c|c|c|c|c|}
\hline CATEGORIA & $\begin{array}{l}\text { SUBCATEGORIAS } \\
\text { PRIMÁRIAS } \\
\end{array}$ & SUBCATEGORIAS SECUNDÁRIAS & $\mathbf{f}$ & $\%$ \\
\hline \multirow{16}{*}{$\begin{array}{l}\text { TD podem } \\
\text { facilitar o } \\
\text { processo de } \\
\text { ensino e } \\
\text { aprendizagem }\end{array}$} & \multirow[t]{14}{*}{ Sim } & Sem justificativa & 41 & 50,0 \\
\hline & & É mais divertido/prático/legal & 11 & 13,3 \\
\hline & & $\begin{array}{l}\text { Facilita estudos, processos, pesquisa, } \\
\text { referências }\end{array}$ & 6 & 7,3 \\
\hline & & Mais interessante, chama mais atenção & 5 & 6,6 \\
\hline & & Mais rapidez e tecnologia portátil & 3 & 3,6 \\
\hline & & $\begin{array}{l}\text { Mais conhecimento, informação, } \\
\text { aprendizagem }\end{array}$ & 3 & 3,6 \\
\hline & & Sai da rotina & 2 & 2,4 \\
\hline & & Estamos acostumados fora da aula & 2 & 2,4 \\
\hline & & Ajuda na escrita/Escrever mais rápido & 2 & 2,4 \\
\hline & & Concentração e pesquisa & 1 & 1,2 \\
\hline & & Gostamos mais & 1 & 1,2 \\
\hline & & Para quando precisamos imprimir & 1 & 1,2 \\
\hline & & Estamos legados à tecnologia & 1 & 1,2 \\
\hline & & Não vai ter preocupação se não copiar & 1 & 1,2 \\
\hline & Não & Sem justificativa & 1 & 1,2 \\
\hline & Mais ou menos & Tem gente que não entende muito bem & 1 & 1,2 \\
\hline Total & & & 82 & 100 \\
\hline
\end{tabular}

Com base no Quadro 2 observa-se que a maioria dos participantes ( $\mathrm{f}=41,50 \%$ ) não justificaram sua resposta; dos que o fizeram, as justificativas de maior frequência foram "É mais divertido/prático/legal" (f=11, 13,3\%) e "Facilita estudos, processos, pesquisa, referências" ( $\mathrm{f}=6,7,3 \%)$. Tendo como base a justificativa de maior frequência observa-se que, na percepção dos alunos, aprender pode ser algo divertido.

Löbler, Pretto e Bolzan (2013) também constataram em sua pesquisa que os estudantes da educação básica acreditam que seja importante a utilização das TD para potencializar o aprendizado, pois estes recursos, segundo as percepções discentes, os motivam mais e os ajudam na concentração. No estudo de Gewehr e Strohschoen (2017), mais da metade dos discentes entrevistados responderam que aprendem com mais facilidade quando as tecnologias estão presentes.

Considerando os dados apresentados neste estudo observa-se uma abertura e receptividade dos discentes ao uso das TD no ensino, o que pouco foi constatado na prática, segundo suas percepções. Tendo em vista este cenário, convém mencionar $o$ que destacam Malheiros, Souza e Peralta (2020), que reforçam a imprescindibilidade da formação continuada dos professores como possibilidade de proporcionar-lhes 0 aprimoramento de seus conhecimentos sobre as potencialidades das TD no ensino.

As mudanças possibilitadas pelas TD podem ser profundas e, muitas vezes, são pouco exploradas; com a flexibilidade que elas oportunizam, pode-se também adequar 
melhor os espaços, tempos e metodologias de ensino, apontando caminhos, motivando e envolvendo mais os alunos [Moran 2012].

\section{Considerações}

Este estudo teve por objetivo investigar e analisar as percepções de alunos dos anos finais do Ensino Fundamental sobre o uso de tecnologias digitais nas atividades de sala de aula. Os dados encontrados por meio da aplicação de um questionário mostraram que, no contexto destes discentes investigados, os professores utilizam pouco as TD em sua prática pedagógica, com frequência rara e, quando o fazem, é por meio do PowerPoint.

Por outro lado, quando questionados se gostariam de utilizar TD nas aulas, todos os alunos (80) responderam que sim e justificaram esta resposta dizendo que as TD são legais, melhoram o aprendizado e representam uma opção diferenciada. Neste caso, quando questionados sobre a preferência de uso, a maioria dos participantes indicou os aplicativos, seguido dos jogos; menos expressivas foram as opções PowerPoint e blogs, que não se sobressaíram como preferências.

Os discentes indicaram, ainda, que acreditam que as TD podem facilitar o ensino e aprendizagem, principalmente por proporcionar uma aula mais divertida, auxiliar as práticas escolares e aumentar seu interesse.

Observando de maneira integral as respostas dos alunos em relação à utilização das TD pelos seus professores e as suas percepções referentes a este uso, verifica-se um contraponto, pois este é um potencial que não está sendo totalmente aproveitado no espaço escolar, uma vez que os dados indicaram o pouco uso dos recursos pelos professores, e, ao mesmo tempo, expressaram o desejo dos estudantes sobre a sua inserção e utilização nas aulas.

Este cenário indica a importância de olhar para a formação dos professores, tanto inicial, quanto continuada, a fim de fornecer-lhes subsídios para a inserção das TD em suas práticas profissionais, e, mais que apenas a inserção, que ela possa ser feita de maneira crítica e com objetivos definidos.

Esta pesquisa apresenta contribuições no que se refere às percepções de alunos dos anos finais do Ensino Fundamental sobre a utilização das TD em sala de aula, ajudando a elucidar as opiniões deste público a respeito da temática. Mais estudos desta natureza devem ser realizados, a fim de levantar as percepções dos educandos em diferentes contextos de ensino e realidades, para então poder, a partir destes resultados, promover práticas mais assertivas com o uso das TD.

\section{Agradecimentos e Apoios}

O presente trabalho foi realizado com apoio da Coordenação de Aperfeiçoamento de Pessoal de Nível Superior - Brasil (CAPES) - Código de Financiamento 001.

\section{Referências}

Almeida, C. M. M., Lopes, L. A. e Lopes, P. T. C. (2015). "Sequências didáticas eletrônicas no ensino do corpo humano: comparando o rendimento do ensino tradicional com o ensino utilizando ferramentas tecnológicas", Acta Scientiae, v. 17, n. 2, p. 466-482. 
Almeida, C. M. M., Costa, R. D. A. e Lopes, P. T. C. (2017). “Análise do desempenho acadêmico e da aprendizagem significativa no ensino superior utilizando as tecnologias digitais", Nuances: estudos sobre Educação, v. 28, n. 1, p. 25-43.

Almeida, C. M. M., Santos, M. J. and Lopes, P. T. C. (2018). "Electronic Didactic Sequences as Teaching Assistance in the Process of Teaching and Learning in Higher Education in Brazil and Portugal”, Acta Scientiae, v. 20, n. 5, p. 1008-1025.

Anastacio, M. A. S. e Voelzke, M. R. (2020). "O uso do aplicativo Socrative como ferramenta de engajamento no processo de aprendizagem: uma aplicação das Tecnologias Digitais de Informação e Comunicação no ensino de Física", Research, Society and Development, v. 9, n. 3. p. 1-13.

Backes, J. L. e Pavan, R. (2014). "As identidades dos alunos em tempos de cultura digital: a percepção dos professores de educação básica", Revista da FAEEBA Educação e Contemporaneidade, v. 23, n. 42, p. 219-227.

Bardin, L. (2011). Análise de conteúdo. São Paulo: Edições 70.

Fuza, A. F. e Miranda, F. D. S. S. (2020). "Tecnologias digitais, letramentos e gêneros discursivos nas diferentes áreas da BNCC: reflexos nos anos finais do ensino fundamental e na formação de professores”, Revista Brasileira de Educação, v. 25, p. $1-26$.

Gewehr, D. e Strohschoen, A. A. P. (2017). "Percepções e hábitos de nativos digitais sobre ensino e aprendizagem com TDICS na escola e em ambientes não escolares", Imagens da Educação, v. 7, n. 2, p. 24-37.

Gomes, V. X. S. S., Marinho, A. M. C. P. e Carneiro, C. (2016). “Jogo digital como estratégia para aprendizagem da anatomia do sistema locomotor humano na perspectiva de discentes do curso de Educação Física", Tecnologias na Educação, ano 8, v. 17, p. 1-11.

Kirschbaum, C. (2013). "Decisões entre pesquisas quali e quanti sob a perspectiva de mecanismos causais", Revista Brasileira de Ciências Sociais, v. 28, n. 82, p. 179193.

Löbler, M. L., Pretto, D. e Bolzan, L. M. (2013). "Percepção dos Alunos a respeito da Inclusão de Tecnologias Digitais no Ensino Público", Novas Tecnologias na Educação, v. 11, n. 3, p. 1-10.

Lopes, P. T. C., Almeida, C. M. M. e Costa, R. D. A. (2014). "Ensino de Ciências através de Tecnologias de Informação e Comunicação: utilizando uma sequência didática eletrônica e um ambiente virtual de aprendizagem”, Acta Scientiae, v. 6, n. 4, p.161-177.

Machado, S. C. (2016). "Análise sobre o uso das tecnologias digitais da informação e comunicação (TDICS) no processo educacional da geração internet", Novas Tecnologias na Educação, v. 14, n. 2, p. 1-10.

Malheiros, A. P. S., Souza, L. B. e Peralta, P. (2020). “Tecnologias Digitais nas aulas de Matemática: um olhar para Diretoria de Ensino de São José do Rio Preto - SP”, Revista Eletrônica de Educação, v. 14, p. 1-20. 
Masetto, M. T. (2013). "Mediação pedagógica e tecnologias de informação e comunicação". In: Moran, J. M., Masetto, M. T. e Behrens, M. A. Novas tecnologias e mediação pedagógica. 21 ed. São Paulo: Papirus.

Morais, P. H., Morais, B. T. e Góis, A. L. (2018). "Tecnologias da informação e comunicação na educação: um estudo nas instituições de ensino pública municipal e estadual de Angicos-RN", Tecnologias na Educação, v. 28, ano 10, p. 1-12.

Moran, J. M. (2012). A educação que desejamos: novos desafios e como chegar lá. 5 ed. São Paulo: Papirus.

Moran, J. M., Masetto, M. T. e Behrens, M. A. (2013). Novas tecnologias e mediação pedagógica. 21 ed. São Paulo: Papirus.

Oliveira, A. X., Mello, D. E. e Franco, S. A. P. (2020). "Práticas de ensino com o uso de tecnologias digitais: o papel da formação docente”, Revista Teias, v. 21., n. 60, p. 7590 .

Panucci-Filho, L., Santos, C. A. e Brito, L. A. (2011). "Vantagens e desvantagens sobre a aprendizagem percebidas pelos alunos de graduação do ensino mediado com o PowerPoint TM: Um estudo exploratório", Revista Iberoamericana sobre Calidad, Eficacia y Cambio en Educación, v. 9, n. 3, p. 94-112.

Pery, L. C., Cardoso, S. P. e Nunes, W. V. (2010). "Jogos educativos digitais: ludicidade e interatividade no ensino nas séries iniciais". In: Congresso Iberoamericano de Informática Educativa, Santiago, Chile.

Piovesan, A. e Temporini, E. R. (1995). "Pesquisa exploratória: procedimento metodológico para o estudo de fatores humanos no campo da saúde pública", Revista Saúde Pública, v. 29, n. 4, p. 318-325.

Santos, V. A., Rodrigues, A. e Junior, M. K. R. (2018). “'Tenho um tablet, e agora?': A produção de narrativas digitais como estratégia na formação de professores de Ciências", Alexandria: Revista de Educação em Ciência e Tecnologia, v. 11, n. 2, p. 31-55.

Sarker, M. N. I., Wu, M., Cao, Q., Alam, G. M. and Li, D. (2019). Leveraging Digital Technology for Better Learning and Education: A Systematic Literatura", International Journal of Information and Education Technology, v. 9, n. 7, p. 453461.

Schuhmacher, V. R., Filho, J. P. A. e Schuhmacher, E. (2017). "As barreiras da prática docente no uso das tecnologias de informação e comunicação", Ciência \& Educação, v. 23, n. 3 , p. 563-576.

Silva, C. C. e Nascimento, R. M. T. (2020). "Informática básica na escola: uma experiência no ensino fundamental”, Brazilian Journal of Develpment, v. 6, n. 4, p. 21668-21676. 